\title{
Anne Douaire-Banny, Remembrances. La nation en question ou L'autre continent de la francophonie
}

\section{Emanuela Cacchioli}

\section{(2) OpenEdition}

1 Journals

\section{Edizione digitale}

URL: http://journals.openedition.org/studifrancesi/4490

DOI: 10.4000/studifrancesi.4490

ISSN: 2421-5856

\section{Editore}

Rosenberg \& Sellier

\section{Edizione cartacea}

Data di pubblicazione: 1 settembre 2016

Paginazione: $369-370$

ISSN: 0039-2944

\section{Notizia bibliografica digitale}

Emanuela Cacchioli, « Anne Douaire-Banny, Remembrances. La nation en question ou L'autre continent de la francophonie », Studi Francesi [Online], 179 (LX | II) | 2016, online dal 01 septembre 2016, consultato il 18 septembre 2020. URL : http://journals.openedition.org/studifrancesi/4490 ; DOI : https://doi.org/ 10.4000/studifrancesi.4490

Questo documento è stato generato automaticamente il 18 settembre 2020.

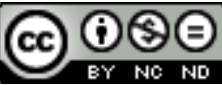

Studi Francesi è distribuita con Licenza Creative Commons Attribuzione - Non commerciale - Non opere derivate 4.0 Internazionale. 


\title{
Anne Douaire-Banny, Remembrances. La nation en question ou L'autre continent de la francophonie
}

\author{
Emanuela Cacchioli
}

\section{NOTIZIA}

ANNE DOUAIRE-BANNY, Remembrances. La nation en question ou L'autre continent de la francophonie, Paris, Honoré Champion, 2014, 354 pp.

1 Come si può ancora usare il concetto di nazione all'interno di una società multilinguistica? E come considerare la nazione nel contesto delle letterature francofone? Possiamo continuare a pensare alla Francia come centro della francofonia? Per rispondere a questi quesiti Anne Douaire-Banny riflette sulla questione della nazione e ne indaga tutti i possibili snodi all'interno dell'ambito fecondo della francofonia. Secondo la studiosa, occorre innanzitutto constatare il rapporto dialettico che si stabilisce tra le letterature francofone e la Francia. Quest'ultima, infatti, per lo scrittore francofono, costituisce da un lato un «réservoir d'idées, de logique, de textes» (p. 32) e, dall'altro, un «repoussoir» (p. 32) a causa dei rapporti di dominio che hanno caratterizzato la relazione politica, militare e simbolica tra colonia e madrepatria. Anne Douaire-Banny vuole dunque comprendere come viene affrontato nelle opere letterarie francofone il sentimento nazionale, sfruttando quest'ultimo come «prétexte du questionnement plus large sur l'altérité» (p. 26).

2 Il volume è suddiviso in tre parti. La prima sezione contiene una riflessione sul rapporto tra desiderio nazionale e possibili derive nazionaliste. Se l'identità nazionale esiste in virtù dei dibattiti che ne mostrano il carattere sacrale o il pericolo latente, è il desiderio che nasce da essa il rischio maggiore. Prendendo in prestito la metafora adottata da Amin Maalouf in Les identités meurtrières, Anne Douaire-Banny paragona questo desiderio a una pantera, ossia a un animale che difficilmente può essere 
addomesticato al punto da divenire animale da salotto. La pantera resiste ai discorsi, è in grado di riformularsi e presenta le medesime insidie anche quando assume forme differenti. Nello specifico la studiosa analizza l'euforia che nasce nelle popolazioni africane dopo il raggiungimento dell'indipendenza. Questa fase lascia ben presto il posto a sentimenti di disillusione e di frustrazione. Tali atteggiamenti si concretizzano in letteratura con un ricorso alla deformazione della realtà, all'uso di iperboli e altri artifici retorici. Questi ultimi consentono di creare personaggi e scene caricaturali, smisurate, capaci di rendere conto della collera, della sete di rivalsa e della violenza latente che hanno permesso di raggiungere l'indipendenza e che ora, nonostante la disillusione, assicurano agli autori la possibilità di credere nella persistenza di una comunità immaginaria a dispetto delle evidenze. Il desiderio nazionale trova, quindi, nuove forme anche in questo contesto di delusione e di disincanto. La studiosa si sofferma proprio sulle modalità creative che permettono la costituzione di queste comunità immaginarie e il ritorno della pantera, intesa come «séduction de la langue qui se révèle dans le secret de la lecture» (p. 116) e che diventa, dunque, speranza di unità e di una ritrovata coesione.

3 Nella seconda parte, la riflessione si sofferma sul valore dei miti fondatori e sulla capacità dell'artista di separarsi dai racconti che postulano la nascita della nazione per crearne di nuovi, più fecondi e meglio aderenti alla realtà. Del resto questi miti non sono più reperibili in molti contesti perché infranti o sovvertiti dall'avvento della colonizzazione. Ma questo non aiuta, perché la popolazione si sente doppiamente orfana, in quanto priva di storie che spieghino le origini del proprio paese e in conflitto con quanto imposto dall'occidente. Nel momento in cui le identità non riescono a definirsi, devono necessariamente ricorrere a una sovrapposizione di tratti culturali e la letteratura diventa il banco di prova delle possibili riconfigurazioni sociali e culturali. Ed è proprio questa dimensione transculturale che va a ricostituire i miti perduti dell'origine e il racconto fondatore di cui necessita la popolazione. La letteratura, quindi, combinando i sintomi e la storia della malattia, mette in atto un processo di identificazione che destruttura i vecchi modelli socio-politici. La letteratura diventa così uno specchio in grado di riprodurre fedelmente la realtà, di mostrarci qualcosa che nessuno aveva ancora visto e di rendere conto, in questo modo, del rapporto complesso che intercorre tra una popolazione e la sua storia e tra autore e storia. La riflessione prosegue con un'analisi della riscrittura delle origini a partire da questa apertura all'alterità che è il postulato di base delle opere di Édouard Glissant. Secondo Douaire-Banny, le dinamiche di relazione permettono di aprire nuove prospettive laddove gli studi postcoloniali le chiudono. Dopo aver esaminato le nozioni di mondialità e mondializzazione, di sistema-mondo e di letteratura-mondo, la studiosa ipotizza l'idea di «alternation» (p. 300), ossia di una concezione di nazione scevra dalle logiche di chiusura, di esclusione e di antagonismo. Se la nazione cessa di essere un assoluto identitario, rompe con la tradizione, con la concezione esclusivamente occidentale e diviene apertura e pluralità.

La terza parte riflette sulla possibilità di creare una storia letteraria della francofonia capace di rinnovare la lettura dei testi e la visione del mondo in essi contenuta. In un primo momento, Anne Douaire-Banny dedica un'analisi ai tentativi di storia della letteratura che hanno già preso come punto di vista la diversità francofona. E si interroga dunque sulle modalità che permetterebbero di coniugare l'unità linguistica all'interno di contesti diversi, sulla possibilità di rendere conto della centralità di Parigi nel mercato dell'editoria e del peso da attribuire al binomio centro/periferia. Dopo 
queste constatazioni preliminari, la studiosa abbozza una storia della letteratura capace di superare la dicotomia centro/periferia e di fondarsi sul rapporto individuo/ comunità all'interno di un sistema-mondo. Il desiderio nostalgico del nazionale e le forme di un'altra nazione possibile «intègrent le passé et l'avenir dans l'instant envisagé comme celui de toute fondation possible» (p. 219). A partire da questa concezione si organizza tutto il sapere sul mondo e una nuova riflessione epica. Nell'ultimo capitolo, Anne Douaire-Banny si occupa proprio dell'«institutionnalisation de l'intuition épique» (p. 220) e indaga le modalità attraverso le quali l'immaginario della relazione diventa cornice di riferimento per il pensiero letterario e la conoscenza del mondo. La storia della letteratura ha, quindi, una dimensione sociologica (trascrive la visione della società da parte di uno o più membri che vi appartengono in quanto condividono la stessa lingua) e antropologica (è il discorso sul rapporto tra uomo e mondo).

5 Nel capitolo conclusivo l'autrice spiega anche il significato del titolo: con remembrances si intende la volontà di costituire un corpus letterario in cui ogni testo rappresenta un elemento da valorizzare e non da reprimere. Lo scopo di questo volume è, quindi, quello di adottare la poetica della relazione di Glissant non più solo come un modello utopico, ma come una politica in grado di aprirsi alla realtà circostante e di costituire un valido supporto critico per leggere qualsiasi testo francofono in una logica di inclusione e non di esclusione. Si tratta, appunto, di un modo per ricostruire quel corpo smembrato che la concezione di nazione in senso tradizionale aveva prodotto.

Il grande pregio di questo volume è la modalità rigorosa con la quale viene condotta la riflessione, dal primo all'ultimo capitolo. La studiosa mostra, inoltre, un grande livello di erudizione perché ogni sua riflessione è sostenuta da citazioni tratte da opere appartenenti alle letterature francofone di quasi ogni parte del mondo. Oltre a Édouard Glissant, suo punto di riferimento indiscusso soprattutto nella seconda e nella terza parte del volume, Anne Douaire-Banny esplora le opere letterarie di Aimé Césaire, Kateb Yacine, Ahmadou Kourouma, Tierno Monénembo, Mohammed Dib, Assia Djebar, Driss Chraïbi, Réjean Ducharme, Henry Bauchau, Nicolas Bouvier. Si tratta di un'ulteriore dimostrazione del fatto che le letterature francofone costituiscono uno spazio privilegiato per spostare la questione del nazionale dalle derive nazionaliste e aprire nuove prospettive di riflessione, grazie al fatto che ogni paese francofono ha una sua dimensione nazionale e, al tempo stesso, intrattiene una relazione di tipo politico, sociale e culturale con la Francia. 